\title{
CURRENT RESEARCH IN MULTIPLE SCLEROSIS
}

\author{
E. J. Field, M.D., M.S., Ph.D., ANd Henry Miller, M.D., F.R.C.P. \\ Department of Neurology, Royal Victoria Infirmary, Newcastle upon Tyne
}

Despite more than a century of study, the cause of multiple or disseminated sclerosis (MS) still eludes us. It is perhaps even more remarkable that current hypotheses of aetiology and pathogenesis, which must furnish a guide to experimental investigation, can be framed only in vague and tentative terms, and that the general nature of the primary pathological process involved remains unknown. At such a stage in our understanding of the disease the incrimination of multiple aetiological factors is not surprising. The validity of some such factors is indubitable. Looking back, however, the investigator cannot fail to recall the irrelevance of similar excellent studies of the geographical, social, and familial incidence of general paresis once the central role of the causal organism had been established.

The student of MS is hampered by other difficulties-by the absence of any clear definition of the disease as a clinical or pathological entity; by its extraordinarily variable prognosis and natural history, which greatly embarrass the assessment of therapy; and especially by the paucity of evidence as to the nature of its initial histopathological lesion. It is this last hiatus which still permits dispute as to whether the massive demyelination of the established disease represents the end-result of an initially inflammatory lesion, or a primarily biochemical process.

A handful of pathologists in Europe ${ }^{24}$ and the United States ${ }^{9,26}$ adheres to the view that MS is a spirochaetal disease. Like most uncommitted workers who have re-examined the evidence in this connection and have prepared sections on the lines suggested, we remain sceptical. Indeed, it has been plausibly suggested that some such 'spirochaetes' represent nothing more than slivers of glass derived from cover-slips. ${ }^{8}$ Less heterodox recent investigators of the disease have usually taken the hypotheses of a viral aetiology or an allergic pathogenesis as their starting point. These hypotheses are, of course, not mutually exclusive.

Our own approach to the problem of MS has been eclectic, in the hope that one or other avenue, $\vec{\omega}$ might yield clues worth more intensive explora- $\bar{C}$ tion. Such an attack has already produced-as 3 indeed it was bound to do-a number of what Arnold Rich has called ' further contributions to $\rightarrow$ the Fournal of Negative Investigations,' while if $\vec{\oplus}$ our clinical researches ultimately yield any defini- $\vec{\sim}$ tive results they will not be apparent for several $\mathscr{\sigma}^{\circ}$ years. Much of what follows is as yet unpub-o lished, and a good deal uncompleted. Nevertheless, a brief conspectus of work undertaken $\rightarrow$ with a succession of collaborators, viewed in its relation to researches proceeding elsewhere, may $\vec{\bullet}$ stimulate thinking about one of the most intriguing problems in modern medicine.

\section{Acute Disseminated Encephalitis and Multiple Sclerosis}

On the principle of proceeding from the known to the unknown, we selected for initial study $\stackrel{\mathbb{Q}}{\varrho}$ those acute demyelinating diseases in which the $\overrightarrow{\overrightarrow{0}}$ main aetiological factor was apparent. The medical $\frac{3}{3}$ records of the Ministry of Pensions were first utilized for a follow-up study of 27 patients who had recovered from encephalomyelitic illnesses $\overline{0}$ following vaccination against smallpox (PVEM).12 Approximately a third of all the cases had died in the illness, but during the subsequent decade no survivor developed multiple sclerosis or any other $ᄋ$ neurological illness of demyelinating type. However, the possible fallacies of such a retrospective 윽 analysis are indicated by a case later described, ${ }^{16} D$ omitted from this service material because of subsequent re-classification, in which Jennerian N re-vaccination had been immediately followed by the onset of classical MS.

A second follow-up study of 27 patients who had recovered from acute disseminated encephalo- $\bar{O}$ myelitis (ADEM) in this hospital between 19320 and 1942 yielded rather similar results. ${ }^{14}$ In con- $\bar{D}$ trast with the findings in several previously $\stackrel{\infty}{+}$ reported series, no case had developed frank MS 0 during an average period of I4 years' observation

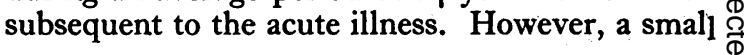


number of recurrences of benign encephalomyelitic type was observed during this period, while one patient manifested a non-progressive syndrome indistinguishable from classical MS.

These observations were taken to confirm the view that while ADEM and PVEM were syndromes easily confused with MS because of diagnostic difficulties, they were nevertheless clinicopathological entities distinct from the chronic disease. Again, further experience has made us less certain of this view, for we have recently encountered two cases in which classical MS was punctuated $^{16}$ or initiated by typical encephalomyelitic illnesses characterized by fever and clouding of consciousness.

At an early stage of our investigations we had been impressed by the analogies which could be drawn between ADEM and the collagen-vascular diseases. Both appeared to follow non-specific incidents, often of an infective nature; in both the pathological changes appeared to be localized in the vicinity of blood vessels; and in each the clinical syndromes produced seemed to depend on the tempo of the pathological process involved, on its localization, and on the calibre of the vessels predominantly affected. It was this analogy which led us to regard ADEM as an "urticaria of the nervous system '11 and to try the effect of corticotrophic hormone, first in $\mathrm{ADEM},{ }^{13}$ and subsequently on a small series of cases of MS in acute exacerbation. ${ }^{15}$ ADEM is a rare disease, and controlled observations were impossible. The results of treatment were suggestive, and on occasion apparently dramatic. Subsequent work has added little in the way of further definition. In a very small series of cases of acute MS our findings appeared to confirm those of earlier observers, ${ }^{6}$ that no definite benefit was seen from this form of treatment.

As is often the case, attempts to assess the efficacy of treatment emphasized our ignorance of the natural history of the untreated syndromes, and we therefore embarked on comprehensive reviews of the neurological sequelae of the acute specific fevers ${ }^{18}$ and of prophylactic inoculation. ${ }^{17}$ These studies confirmed the general similarity of the encephalitic, myelitic, and polyradicular (Guillain-Barré) syndromes seen in these various clinical contexts. Certain differences were seen within the series, for example the tendency of varicella to produce a milder encephalitis than measles or rubella, with a higher incidence of cerebellar signs; and the predominantly central incidence of occasional neurological complications of T.A.B. inoculation, as compared with the radicular involvement typical of reactions to the parenteral administration of horse serum. Nevertheless, the generally unfavourable prognostic significance of deep coma, convulsions, and hemiplegia, and the good outlook for functional recovery in transverse myelitis, as well as the ubiquitous histopathological signature of perivenous oedema, infiltration, and demyelination suggested the involvement of a common pathogenetic factor which, with Glanzmann, ${ }^{5}$ we took to be in all probability some form of tissue hypersensitivity. Interesting as these findings were, they did little to illumine the central problem of MS, or to point the direction of future progress when in 1956 we found ourselves adequately financed by local enterprise to devise a programme of clinical investigation into this disease.

\section{The North-East Multiple Sclerosis Survey}

Our first step was to undertake a clinical survey of MS in Northumberland and Durham. This was essentially a case-finding survey, epidemiological consideration being secondary. Indeed, it has been held that the incidence of the disease is of an order which renders a definitive assessment of prevalence all but impossible. Intensive survey of a population of manageable size would yield too few cases to furnish a basis for statistical comparison, while valid figures could be obtained only from a population so large as to render detailed scrutiny impracticable.

In this survey a population of approximately I,400,000, registered with $35^{2}$ general practices (out of a total of 519 in the two counties), furnished 662 authenticated cases of MS-a prevalence rate of 47 per 100,000 of the total population at risk. A prevalence figure of 59 per 100,000 was yielded by a smaller population of just under half a million registered with practitioners personally known to us for the care with which they kept their records and their readiness to refer patients for specialist opinion. The higher figure is likely to be the more accurate, though still certainly an underestimate, and it implies the presence of not less than $\mathrm{I}, \mathrm{I} 00$ established cases in the area.

All 662 cases traced were personally examined and fully recorded by Dr. Kurt Schapira. The clinical data accumulated awaits analysis, and is being used first to answer some simple questions about the natural history of the disease. It has already been shown by analysis of a large series of consecutive cases that lumbar puncture has little or no effect on the course of the disease, ${ }^{21}$ and from a similarly controlled study much the same seems to be true of surgical operations carried out on patients with MS. A further study based on this material has shown that there is no evidence of any differential seasonal incidence of onset or exacerbation, ${ }^{22}$ a finding of some interest since a 
higher prevalence in the colder parts of the world is one of the few epidemiological facts about the disease which seems reasonably established. In a fourth study, initial results suggest that MS may be proportionately commoner in the RegistrarGeneral's Social Class I and rarer in Social Class V than would be expected-in other words that it is possibly a disease of relative affluence. There may, however, be a statistical fallacy in this analysis, and a random sample of cases drawn from that part of the population excluded from the initial survey because of the non-co-operation of some doctors is at present being examined. Familial incidence and the influence of pregnancy on the course of the disease are to be amongst the subjects of further studies.

\section{Gamma-Globulin Studies}

With the collaboration of Miss D. C. Park, working in the Department of Chemical Pathology, paper electrophoresis using a reflectance densitometer has been employed to estimate gammaglobulin content ${ }^{1,10,27}$ in the spinal fluid and serum of $8 \mathrm{I}$ patients with $\mathrm{MS}, 5^{\mathrm{I}}$ with other neurological disorders, and 25 control cases considered free of structural nervous disease. No significant differences were found between the serum gamma-globulin levels in the various groups, but in the spinal fluid the level of gammaglobulin expressed as a percentage of total protein was found to be significantly raised above the upper level of the mean (14.28 per cent.) in 85 per cent. of all cases of MS. Unfortunately I6 per cent. of normal controls and no less than 38 per cent. of patients with other neurological disorders also gave figures above this level. In other words, although the increase in gammaglobulin in MS is highly significant statistically, it is of limited value in differential diagnosis. The extension of these observations to other neurological disorders may resolve present uncertainty as to whether the increase in gammaglobulin content implies an immunological disturbance, or is merely a sequel to the destruction of myelin.

\section{Therapeutic Trials}

Patients traced by the survey have also been used in three therapeutic trials, designed with the statistical advice of Mr. D. J. Newell of the Nuffield Department of Industrial Health.

The first of these involves an attempt to discover whether the natural history of MS is influenced by the intrathecal administration of tuberculin (P.P.D.) on the lines suggested by Honor Smith. ${ }^{25}$ Ninety moderately disabled cases are being randomly distributed amongst three groups treated under similar conditions withڤ (I) intrathecal P.P.D. and intensive physiotherapy, 3 (2) intrathecal saline and intensive physiotherapy, $₫$ (3) intensive physiotherapy alone. Sixty patientsc. have so far been treated. We have confirmed $\Rightarrow$ Smith's finding that the administration of intra- $\frac{\rho}{+}$ thecal tuberculin may provoke an exacerbation of $\bar{C}$ MS, but we cannot yet assess any possible value $\frac{\bar{\sigma}}{\bar{c}}$ the procedure may have in preventing relapse or $\frac{\text { D }}{\bar{\sigma}}$ in arresting deterioration. Disability is being $\odot$ periodically measured on the basis of a simple scoring system, together with serial cinematograph $\vec{\circ}$ records of standard performance tests. An interim: assessment will be possible in November 1960, $\vec{\omega}$ but final results not before 1962 and 1965 .

The second experiment involves a 'doubleblind' two-year trial of maintenance dosage with prednisone, salicylate, and dummy tablets in three $\omega_{\sigma}^{\omega}$ groups each of 30 ambulant MS patients to $A$ observe any apparent effect on relapse-rate or $\vec{f}$ deterioration of function.

The final observation of this kind comprises the admission and comprehensive clinical investi-을 gation of patients with acute exacerbations of MS in an attempt at the controlled assessment of the $\frac{D}{0}$ efficacy of treatment with corticotrophic hormone in this acute phase. The therapeutic trial is onty $\vec{\varnothing}$ one part of this investigation, which seeks also elucidate other features of acute exacerbation How often can provoking factors be identified? What is their nature? Is there any clinicopathological evidence of visceral or humoral disturbance in these episodes? This study is being $\frac{\circ}{\Phi}$ combined with a search for antibodies to nervous and other tissues in the blood and spinal fluid of these and other cases by a variety of techniques. In this we have had the collaboration of Dr. S. G. Owen of the Department of Medicine, and Dr. A. L. Latner of the Department of Chemical 윽 Pathology. In a proportion of acute exacerbations of MS, antibodies to human nervous tissue have 3 been detected in the patients' serum, but their inconstancy (they are rarely found in quiescent $\stackrel{3}{\xi}$ cases) suggests that they may possibly represent 0 merely an over-spill of antibody which is mostly fixed to nervous tissue. Moreover, no patho- $\frac{D}{0}$ genetic significance can be attached to antibodies to myelin in MS until we can be sure that they of represent more than a result of myelin degenera- $N$ tion. As in the case of gamma-globulin, a parallel $N$ study of ' degenerative' diseases characterized by myelin destruction is imperative.

It is unfortunate that though we now have close on a thousand MS patients on our books, $\stackrel{\Phi}{\oplus}$ and although we circularize practitioners in the region unceasingly, we admitted in $195^{8}$ only 13 cases of exacerbation which satisfied our criteria for inclusion in the therapeutic trial. 


\section{A Practice of Anaesthesia}

\author{
by W. D. WYLIE
}

M.B.(Cantab.), M.R.C.P.(Lond.), F.F.A.R.C.S.

Consultant Anaesthetist, St. Thomas's and other Hospitals

H. C. CHURCHILL-DAVIDSON

M.D.(Cantab.), F.F.A.R.C.S.

Consultant Anaesthetist, St. Thomas's and other Hospitals

\section{Practical Obstetric Problems} by IAN DONALD

M.B.E., M.D.(Lond.), F.R.F.P.S.(Glas.), F.R.C.O.G.

Regius Professor of Midwifery, University of Glasgow

Fluid Balance in Obstetrics $x v i+1056 p p$.

327 illustrations,

1 colour plate

(1960) 105s. net

$x v i+712 p p$.

139 illustrations

(1959) 55s. net

(2nd. edit.)

$x+170 p p$

5 illustrations

(1960) 25s. net

$v i i i+184 p p$.

(1960) 10s. net

(10th edit.)

Physician Superintendent, Ruchill Fever Hospital, Glasgow

49 Newman Street, London, W.

\section{Periphlebitis Retinae}

Partial optic atrophy is a familiar clinical sign of MS, but there has been a number of recent reports, especially from Scandinavia ${ }^{7}$ and Hungary, ${ }^{19}$ describing ' sheathing' of the small peripheral retinal veins in this disease, the suggestion being that these visible retinal changes may represent a form of ' periphlebitis nodosa,' perhaps provoked by an inflammatory or allergic mechanism, which might be present also throughout the neuraxis. Here the absence of lymphatic drainage might further impair absorption of spinal fluid and provoke perinevous oedema and subsequent demyelination. In view of this suggestion that the retinal changes might be a valuable pointer to the pathogenesis of the disease in general, a study of the accessible retina is being undertaken, using a Zeiss retinal camera.

In none of the 14 cases of multiple sclerosis so far examined has anything resembling these changes been found. Sometimes a glistening veillike appearance is encountered in the neighbourhood of the retinal veins which bears some resemblance to published drawings, but which is certainly an artifact in that it depends on a certain obliquity of lighting. These ' reflexes' may possibly be more common in multiple sclerotic fundi than in those of normal control patients, but evidence as yet is inconclusive.

\section{Immunological Observations in Patients with Multiple Sclerosis}

In view of the current suspicion that allergic factors may be involved at some stage in the pathogenesis of MS it was thought worth while to investigate the antibody-producing power of these patients as compared with normal subjects. The lack of facilities to study immunization against tetanus, which we would have regarded as the procedure of choice, led us to observe the response to T.A.B.T. inoculation as assessed by routine hospital Widal tests carried out in the Department of Bacteriology. It was thought that MS patients might possibly show an abnormally reactive antibody response, or alternatively that their preoccupation with the pathogenesis of the neurological disorder might lead to a diminished reaction.

The response observed in 20 mobile MS patients was compared with that seen following their routine T.A.B.T. inoculations in 20 mentally defective subjects to whom access was kindly allowed by Dr. G. McCoull of Prudhoe and Monkton Hospital. A Widal test was carried out 
on all subjects before the beginining of the experiment, a fortnight after the first inoculation, and simultaneously with further T.A.B.T. injections given at intervals during succeeding months.

These observations yielded results of interest from two points of view. First, they furnished suggestive evidence that the immediate response to T.A.B.T. inoculation is much less marked in MS than in control cases, though no ultimate differences were observed between the two groups in the titres of $\mathrm{TO}, \mathrm{TH}, \mathrm{BO}, \mathrm{BH}, \mathrm{CO}$, nonspecific and Brucella agglutinations. Secondly, they showed that even in normal subjects the immunological response indicated by Widal testing was surprisingly small, fluctuating and illsustained, a finding in keeping with the doubts raised by Cvjetanovic ${ }^{4}$ as to the prophylactic efficacy of certain typhoid vaccines in common use.

\section{Virus Studies}

Predominant involvement of white matter and relative sparing of the neurones has usually been accepted as indicating that the origin of MS in invasion by a specific virus was rather improbable.

Claims that demyelination can occur as a prominent and direct effect of invasion of the nervous system must be treated cautiously. In murine encephalitis (JHM virus), for example, myelin destruction would appear to be secondary to necrosis of brain parenchyma; Sigurdsson's work on Visna $^{23}$ claiming as it does that a chronic demyelinating disease of Icelandic sheep may be experimentally transmitted to other animals by intracerebral injection of a cell-free filtrate, awaits independent confirmation; whilst the occasional demyelination of canine distemper may be a secondary. phenomenon akin to that of human ADEM (e.g. complicating measles).

Virus studies have recently been carried out on material obtained four hours after death from the brain of a young woman who died from medullary failure during an uncomplicated acute episode of unequivocal MS. Culture in Hela cells, embryonic rabbit kidney, and chorioallantoic membrane, as well as inoculation of a variety of animals has proved negative in two different laboratories, while intracerebral inoculation into sheep has similarly so far failed to produce positive changes clinically or in serial spinal fluid examinations. It is of some interest that in our hands the normal cell count in the spinal fluid of the sheep (six animals) has not exceeded i i cells per ml., while Sigurdsson regards figures of up to 50 cells as normal. The possibility of latent infection in 'normal' sheep must be kept in mind, and the need for this work to be repeated on a larger scale has led us to enlist the help of Dr. J. P. Stamp of the Moredun Institute in Edinburgh, who is carrying out a more extensive trial with a preparation of the气 same pathological material.

\section{Experimental 'Allergic' Encephalitis}

In September 1958 the timely generosity of the Leverhulme Trustees permitted us to complemento our clinical observations by a programme of $\frac{\overline{\bar{D}}}{\overline{\mathrm{C}}}$ experimental studies.

Since Rivers and Schwentker's ${ }^{20}$ demonstration that a demyelinating encephalomyelitis can be produced in monkeys by repeated injections of $-\vec{O}$ brain emulsion, and the subsequent observation:that its development can be facilitated and ac- $\vec{\omega}$ celerated-though remaining otherwise apparently unchanged - by combining the emulsion witho ' adjuvants,' experimental ' allergic' encephalomyelitis (EAEM) has been widely employed as a research tool by investigators of the demyelinating $A$ diseases. It has proved of great value in studying $A$ the experimental pathology of the nervous system, though it is to go beyond the facts to regard ${ }^{\circ}$ EAEM as in any sense an authentic model of MS. $\frac{\text { S }}{9}$ However, if we tentatively accept a qualitative $\overrightarrow{-}$ relationship between MS and human ADEM, theo histopathological appearances of the latter certainly resemble those of EAEM, at any rate in the $\vec{\varnothing}$ monkey, where perivascular demyelination is often? conspicuous. It must be admitted that in the smaller animals usually employed in studies of EAEM perivascular infiltration is much more in evidence than demyelination, but the pathogenetic. mechanisms involved are almost certainly similar.

We have studied a series of drugs and proce- $-\overrightarrow{\vec{B}}$ dures which might be expected to militate against 3 the development of allergic processes involved in the evolution of EAEM. Sodium salicylate is more effective in preventing EAEM in the guineapig when given intraperitoneally than when it is 3 injected subcutaneously, a difference possibly re-; lated to the irritant effect of the former procedure, 3 . which represents a substantial stress. Corticotrophin more effectively suppresses the clinical signs of EAEM and reduces its histological in-o tensity. Little information is available about the tolerance of guinea-pigs to $X$-irradiation but pre-o liminary experiments showed that 900r was regularly fatal within eight or nine days, whiles even a single dose of 3 oor led to a 50 per cent. N mortality. X-irradiation ( 15 or or 3 oor 18 to $24 \mathrm{~N}$ hours before encephalitogenic inoculation) did not inhibit the development of EAEM. However, since many animals develop skin sensitivity despite heavy irradiation, these results cannot be taken as compelling evidence against the allergic 0 nature of the experimental disease. It would ${ }^{\circ}$ seem that in the guinea-pig even lethal or sub- $\mathbb{\Phi}$ lethal doses of X-rays do not wholly suppress $\underset{\mathbb{Q}}{\mathbb{Q}}$ 
immunological response to an antigen injected 18 to 24 hours later.

Delayed tuberculin-type skin sensitivity has been studied by measuring the thickness of the skin at a specific point before and at intervals after the intradermal injection of $0.1 \mathrm{ml}$. of a $I$ in 100 brain suspension. The results, expressed as a percentage increasē of thickness, are not highly accurate, but serve for comparative purposes. It has been found that all injected animals develop skin sensitivity to human brain emulsion regardless of whether or not they subsequently develop EAEM; that this begins as early as the second or third day; and that it can be diminished by corticotrophin administration, and to a lesser degree by salicylate. Animals injected with brain emulsion do not develop sensitivity to homologous liver emulsion; nor can skin sensitivity to homologous liver or human brain emulsion be produced by injection of homologous liver plus adjuvants. Furthermore, there seems to be no correlation between skin sensitivity, the presence of circulating antibody (determined by S. G. Owen using the tanned red-cell technique with specimens of blood obtained by cardiac puncture) and the development of EAEM.

Finally we have investigated the claims of Behar and $\mathrm{Tal}^{2}$ that specific changes are found in the liver in EAEM. We have found the changes described by these authors in a comparable proportion of 'normal' animals. Nor has it been found possible to produce EAEM with liver emulsions plus adjuvants. This investigation was undertaken on the supposition that organs other than brain might be the source of antibodies producing demyelination, but the evidence so far lends no support to this idea.

\section{Future Work}

While we cannot share the optimism of some of our American colleagues that an epidemiological approach is likely of itself to solve the problems of MS, we deplore its frequent dismissal as ' nose-counting.' It may provide leads to more intensive investigation, and at the least it furnishes data with which any acceptable hypothesis of aetiology must be compatible. Although it demands little in the way of equipment, it is prodigal of time and money. If resources are available we have four survey projects in mind: (I) An intensive prevalence survey to be carried out in Darlington (pop. 76,000) with practitioner collaboration and using a number of other welldefined diseases (e.g. thyrotoxicosis, amyotrophic lateral sclerosis) as 'markers.' We feel that a unit of this size might avoid some of the difficulties to which attention has been drawn earlier in the paper, and that it could provide figures of comparative value. (2) A prevalence and incidence survey to be carried out with the collaboration of the College of General Practitioners, using groups of interested doctors in various parts of the country who would regard MS as a 'notifiable' disease for a 12-month period. (3) Utilization of our present clinical material for an extensive survey of blood groups in a search for linkage and other genetic factors. (4) A three- to five-year prospective clinical and pathological survey of all cases of ' encephalitis' occurring in the area, with long-term follow-up study.

In the experimental field we have in mind studies of mortality rate in animals given corticotrophin later in the evolution of EAEM, in an attempt to parallel the human therapeutic situation; and further attempts to produce the disease with protein extracts of spinal cord. However, our main efforts in the immediate future will be directed towards investigating the pathogenesis of demyelination. It seems to us that whereas circulating antibodies may prove to be of theoretical interest, or even ultimately of diagnostic importance, fixed or sessile antibodies are probably of greater significance, and we hope to investigate these by the method of fluorescent microscopy developed by Coons and his colleagues, ${ }^{3}$ using pharmacological and other procedures directed to the detachment of sessile antibodies. If demyelination is a sequel of persisting antigen-antibody union, the mobilization of fixed antibody might conceivably have therapeutic implications.

\section{REFERENCES}

I. BAUER, H., and HEITMAN, R. (1958), Deutsch. Z.Nervenheilk, $178,47$.

. BEHAR, A. J., and TAL, C. (1959), f. Path. Bact., 77, 59 1.

3. COONS, A. H. (1957), Ann. N.Y. Acad. Sci., 69, 658.

4. CVJETANOVIC, B. B. (1957), Amer. F. publ. Hith, 47, 578.

5. GLANZMANN, E. (1927), Schweiz. med. Wschr., 8, I45.

6. GLASER, G. H., and MERRITT, H. H. (I952), F. Amer. med. Ass., 148, 898 .

7. HAAR, M. (1953), Acta psychiat. scand., 28, 175.

8. HOFMANN, A., and SCHALTENBRAND, G. (1959), Munch. med. Wschr., 101, 1589.

9. ICHELSON, R. R. (I957), Proc. Soc. exp. Biol. (N.Y.), 95, 57.

ro. KABAT, E. A., MOORE, D. H., and LANDOW, H. (I942), f. clin. Invest., 21,571 .

II. MILLER, H. G. (I95I), Proc. roy. Soc. Med., 44, 852.

12. MILleR, H. G. (1953), A.M.A. Arch. Neurol. Psychiat.,

13. MILLER, H. G. (1953), Brit. med. F., i, 177.

14. MILLER, H. G., and EVANS, M. J. (1953), Quart. F. Med.,

15. MILLER, H. G., and GIBBONS, J. L. (1953), Brit. med. f., ii, I345.

16. MILLER, H. G., and SCHAPIRA, K. (1959), Ibid., i, 737, 81 r. 17. MILLER, H. G., and STANTON, J. B. (1954), Quart. $\mathcal{F}$. Med., 23, I.

18. MILLER, H. G., STANTON, J. B., and GIBBONS, J. L. (1956), Ibid., 25, 427.

19. ORBAN, T. (1955), Ophthalmologica (Basel), 130, 387.

20. RIVERS, T. M., and SCHWENTKER, F. F. (I935), f. exp. Med., 61, 689.

21. SCHAPIRA, K. (1959), F. Neurol. Neurosurg. Psychiat., 22, 238. 22. SCHAPIRA, K. (I959), Ibid., 22, 285.

23. SIGURDSSON, B., and PALSSON, P. A. (1958), Brit. $\mathcal{F}$. exp. Path., 39, 519 .

24. SIMMONS, H.' C. T. (1958), Dtsch. med. Wschr., 83, 1196.

25. SMITH, H. V., ESPIR, M. L. E., WHITTY, C. W. M., and RUSSELL, W. R. (1957), $\dot{\mathcal{F}}$. Neurol. Neurosurg. Psychiat., 20, $\mathrm{I}$.

26. STEINER, G. (1952), F. Neuropath. exp. Neurol., I1, 342.

27. YAHR, M. D., GOLDENSOHN, S. S., and KABAT, E. A. (1954), Ann. N.Y. Acad. Sci., 58, 613. 